\title{
Subtropical Gyre
}

$5 \quad$ Running title: Diel metabolites reflect marine microbial activity

6 Authors: Angela K. Boysen ${ }^{1}$, Laura T. Carlson ${ }^{1}$, Bryndan P. Durham ${ }^{2}$, Ryan D. Groussman ${ }^{1}$,

7 Frank O. Aylward ${ }^{3}$, François Ribalet ${ }^{1}$, Katherine R. Heal ${ }^{1}$, Edward F. DeLong ${ }^{4}$, E. Virginia

$8 \quad$ Armbrust $^{1}$, Anitra E. Ingalls ${ }^{1 *}$

9 Affiliations:

$10{ }^{1}$ School of Oceanography, University of Washington, Seattle, WA, USA. ${ }^{2}$ Department of

11 Biology, Genetics Institute, University of Florida, Gainesville, FL, USA. ${ }^{3}$ Department of

12 Biological Sciences, Virginia Tech, Blacksburg, VA, USA. ${ }^{4}$ Daniel K. Inouye Center for

13 Microbial Oceanography: Research and Education (C-MORE), University of Hawaii, Honolulu,

14 HI, USA

15 * Corresponding author: aingalls@uw.edu; (206) 221-6748; Box 355351, School of

16 Oceanography, University of Washington, Seattle WA 98115

17

18 Competing interests: The authors declare no conflict of interest. 


\section{Abstract}

Light is the primary input of energy into the sunlit ocean, driving daily oscillations in metabolism of primary producers. The consequences of this solar forcing have implications for the whole microbial community, yet in situ measurements of metabolites, direct products of cellular activity, over the diel cycle are scarce. We evaluated community-level biochemical consequences of diel oscillations in the North Pacific Subtropical Gyre by quantifying 79 metabolites in particulate organic matter in surface waters every four hours over eight days. Total particulate metabolite concentration peaked at dusk, even when normalized to biomass periodicity. Osmolytes exhibited the largest diel oscillations, implying rapid turnover and metabolic roles beyond cell turgor maintenance. Metatranscriptome analysis revealed the taxa involved in production and consumption of some estimates. The concentrations of $70 \%$ of individual metabolites exhibited 24 -hour periodicity. Despite the diverse organisms that use them, primary metabolites metabolites, including the osmolyte trehalose. This compound displayed the largest

\section{Introduction}

Light is a powerful forcing on metabolism in the surface ocean, acting at the molecular 
43 level to drive global biogeochemical cycles(1). In surface waters near Station ALOHA (22

$\left.4445^{\prime} \mathrm{N}, 158^{\circ} \mathrm{W}\right)$ in the North Pacific Subtropical Gyre (NPSG), the marine plankton community

45 responds to diel forcing, either directly or indirectly, as demonstrated by daily oscillations in

46 particulate organic carbon (POC)(2), cell division(3), gross primary production, net community

47 production(4), grazing(5), viral infection(6), and nitrogen fixation(7). Genes associated with a

48 wide variety of cellular processes also exhibit diel oscillations in transcript abundance, reflecting

49 the capture of light energy and its conversion to chemical energy during daylight, a process that

50 fuels metabolism over a 24 hour period(8-12). Temporal partitioning of anabolism and

51 catabolism creates diel patterns in the macromolecular composition of the community(12-15). Though most POC in the surface ocean is made up of macromolecules(16,17), the suite

53 of small molecules $(<800$ daltons) produced within cells helps shape the internal and external

54 chemical environment of the plankton community. Small molecules are likely responsible for

55 setting dependencies among different taxa, yet an inventory of these compounds and the

56 plasticity of their concentrations remain largely unknown(18). Comprehensive measurements of

57 intracellular small biomolecules, or metabolites, present in marine microbial communities are

58 scarce and the suite of compounds detected is strongly biased by the methods employed(19).

59 Small polar molecules, in particular, are rarely measured although they are the main component

60 of the aqueous cytosol. Intracellular metabolite profiles of model marine microbes are taxon-

61 specific and respond to environmental perturbations, including diel oscillations in available

62 light(20). Many of these metabolites have not yet been measured in plankton communities and

63 some are without annotated biosynthetic or catabolic pathways(21-27). Thus, a comprehensive

64 inventory of intracellular metabolites will facilitate a deeper understanding of marine microbial

65 physiology and interactions that drive ecosystem diversity and activity $(28,29)$. 
Here we measured particulate metabolite concentrations in samples collected from

67 surface waters near Station ALOHA during eight diel cycles. These data provide an inventory of

68 metabolites in the oligotrophic surface ocean and show the metabolic consequences of the diel

69 cycle. We paired observations of metabolites with gene expression data, POC, and flow

70 cytometry (FCM) measurements. We inferred community physiology over the diel cycle to

71 predict how environmental conditions produce a particular chemical environment within natural

72 populations of marine plankton.

We find that the molar concentration of $70 \%$ of our targeted metabolites oscillate with

74 24-hour periodicity, reflecting large scale community synchrony. Our analysis identifies diel oscillations in compounds that play important roles in managing light-induced redox reactions

76 and biosynthesis of building blocks and energy stores. These compounds are ultimately conduits

77 of energy and nutrients through the microbial ecosystem as they are exchanged between diverse

78 organisms, with repercussions for community diversity and function(30-32). By pairing

79 metabolite data with metatranscriptomes, we identify potential metabolic strategies that

80 organisms deploy for coping with redox oscillations induced by an oscillating energy supply.

81

82 Materials and Methods

83 Sample Collection

84 Samples were collected on the R/V Kilo Moana in the NPSG (near $24.5^{\circ} \mathrm{N}, 156.5^{\circ} \mathrm{W}$ )

85 every four hours for two sampling periods in summer 2015 (period one: July 26, 6:00 - July 30,

86 6:00; period two: July 31 18:00 - August 3, 18:00). To limit variability unrelated to solar

87 forcing, we conducted Lagrangian sampling following two drifters in an anticyclonic eddy(7).

88 Samples for particulate metabolites and transcripts were collected from $15 \mathrm{~m}$ water depth using 
89 Niskin bottles attached to a conductivity, temperature, depth array (CTD). Ancillary

90 measurements for nutrients and heterotrophic bacterial abundance (reported in Wilson et. al.

91 2017) were collected and analyzed with standard Hawaii Ocean Time-series protocols

92 (http://hahana.soest.hawaii.edu/index.html).

93

94 Bulk and taxa-specific carbon biomass

POC concentrations were derived from particulate beam attenuation at $660 \mathrm{~nm}$, as in

96 White et al.(2). Particle absorption was calibrated against discrete POC samples taken near dawn

97 and dusk. Discrete POC and particulate nitrogen (PN) samples were collected by filtration of the

98 ship's underway flow through seawater onto combusted GFF filters. Analysis is described in the

99 supplemental methods.

100

Continuous underway flow cytometry (SeaFlow)(33) was used to count Prochlorococcus,

101 Synechococcus, picoeukaryotes (eukaryotic phytoplankton 2-4 $\mu \mathrm{m}$ in size), and Crocosphaera.

102 These data were supplemented with discrete flow cytometry sample analysis as in Wilson et

103 al.(7). Cell diameters of individual cells were estimated from light scatter by the application of

104 Mie theory to a simplified optical model and converted to carbon quotas assuming spherical

105 particles, as described in Ribalet et al.(34). Carbon biomass was estimated by multiplying cell

106 abundance by carbon quotas.

107

108 Metabolite extraction, data acquisition, and processing

109

Metabolite samples were collected in triplicate at each time point by filtering $3.5 \mathrm{~L}$ of

110 seawater onto a $47 \mathrm{~mm} 0.2 \mu \mathrm{m}$ PTFE (Omnipore) filter using a peristaltic pump, polycarbonate

111 filter holder, and Masterflex PharMed BPT tubing (Cole-Parmer). Filters were frozen in liquid 
112 nitrogen immediately after filtration and stored at $-80^{\circ} \mathrm{C}$. Metabolite extractions employed a

113 modified Bligh-Dyer method(19,27,35), resulting in aqueous and organic soluble metabolites

114 with isotope-labeled extraction and injection internal standards added to both fractions (Table

115 S1, Supplemental Methods). Unused filters served as methodological extraction blanks.

116

117

118

119

120

121

122

123

124

125

126

127

128

129

130

131

132

133

134

Metabolomics data were collected by paired liquid-chromatography mass-spectrometry

(LC-MS) using both hydrophilic liquid interaction chromatography and reversed phase

chromatography with a Waters Acquity I-Class UPLC and a Waters Xevo TQ-S triple

quadrupole with electrospray ionization in selected reaction monitoring mode with polarity

switching, targeting over 200 compounds(19). The software Skyline was used to integrate LC-

MS peaks(36) and data were normalized using best-matched internal standard normalization(19).

A subset of this data are presented in Durham et al. (2019) and Muratore et al. (2020)(14,27).

Metabolites with isotopologue internal standards were quantified in all samples (Table

S1). Trehalose, sucrose, and 2,3-dihydroxypropane-1-sulfonate (DHPS) were quantified with

standard additions. For all other metabolites, concentration $\left(\mathrm{pmol} \mathrm{L}^{-1}\right)$ was calculated from

injections of known concentrations of authentic standards in both water and a representative

matrix to correct for ion suppression. Dimethylsulfoniopropionate (DMSP) loss is known to

occur during methanol-based extractions so concentrations are considered a minimum

estimate(37). Details are in the supplemental methods.

\section{Metatranscriptome data acquisition and processing}

Whole community transcript data are referred to here as prokaryotic transcript data, as

they were enriched in bacterial and archaeal RNA. These metatranscriptome samples were collected on $0.2 \mu \mathrm{m}$ filters simultaneously with the metabolomic data reported here, as 
135 previously reported in Wilson et al.(7) and Aylward et al.(6). The metatranscriptome sequence

136 reads were quality trimmed, end-joined, mapped, and quantified with molecular standards.

137 Metatranscriptome sequence reads were mapped to the ALOHA gene catalog(38) using LAST v

$138959(39)$, and transcript count normalization, leveraging the molecular standards described in

139 Gifford et al.(40). Sequence reads were summed if assigned to the same taxonomic order and

140 Kyoto Encyclopedia of Genes and Genomes (KEGG) orthologue(41).

141 Poly-A+ selected transcript data (referred to here as eukaryotic transcript data) are from

142 the metatranscriptomes presented in Durham et al.(27). These samples were collected on $0.2 \mu \mathrm{m}$

143 filters concurrently with the metabolomic samples and include only the first sampling period.

144 Quality-controlled short reads were assembled using Trinity de novo transcriptome assembler

145 version 2.3.2(42). Using DIAMOND v 0.9.18(43), assembled contigs were aligned to a reference

146 sequence database of marine organisms (MarineRefII reference database,

147 http://roseobase.org/data/, with additions listed in Table S2). Taxonomy was assigned with

148 DIAMOND by using the top $10 \%$ of hits with e-value scores below $10^{-5}$ to estimate the Lowest

149 Common Ancestor of each contig. We assigned putative function using hmmsearch (from

150 HMMER 3.1b2(44), minimum bitscore 30) to find the best-scoring KEGG gene family from

151 KOfam (ver. 2019-03-20) (45). Contig abundances were quantified by mapping the paired reads

152 to the assemblies with kallisto(46). Sequence reads assigned to the same taxonomic group and

153 KEGG ortholog were summed and normalized to the total read pool of the taxonomic group.

154 Details are provided in the supplemental methods.

Metabolites and transcripts were associated with one another using the KEGG database

156 as a scaffold to match metabolites with transcripts coding for enzymes that directly use or

157 produce those metabolites. The R package KEGGREST(47) was used to access the KEGG 
158 database followed by manual curation of these matches.

164 each significantly oscillating signal, the time of peak abundance was estimated by fitting a

165 periodic function (supplemental methods), though we recognize the precision of these peak times

166 is limited by our sampling resolution. Diel periodicity in metabolites was identified for the two

167 different sampling periods independently and jointly. incubators with a 12:12 light:dark cycle. Samples for metabolomics were collected by gentle

172 filtration onto $0.2 \mu \mathrm{m}$ Durapore filters using combusted borosilicate filter towers. Crocosphaera 173 watsonii strain WH8501 was grown at $27^{\circ} \mathrm{C}$ with $50 \mu \mathrm{mol}$ photons $\mathrm{m}^{-2} \mathrm{~s}^{-1}$ in $\mathrm{YBC}$-II artificial 174 seawater medium(50) supplemented with $0.9 \mathrm{mM}$ nitrate; cells were collected just before the 175 lights turned on and just after the lights turned off during exponential phase. Cells were 176 enumerated via a Beckman Z2 Coulter Counter. Prochlorococcus MIT1314 (HLII clade(51)) 177 were grown at $20^{\circ} \mathrm{C}$ with $20 \mu \mathrm{mol}$ photons $\mathrm{m}^{-2} \mathrm{~s}^{-1}$ in Pro99 media(52) prepared with Turks 178 Island Salt Solution and supplemented with $6 \mathrm{mM}$ sterile sodium bicarbonate and $1 \mathrm{mM} \mathrm{N}$ 179 Tris(hydroxymethyl)methyl-3-aminopropanesulfonic acid(53). Prochlorococcus cells were 180 collected 6 hours into the light period during exponential phase and enumerated using the flow 
181

182

183

184 Results

185

186

187

188

189

190

191

192

193

194

195

196

197 198 during the first sampling period.

199

200

201

202

203

\section{Metabolite Inventory}

cytometer BD Influx cell sorter. Axenicity of Prochlorococcus cultures was verified regularly with SYBR-staining and FCM and plating on bacterial $1 \frac{2}{2}$ YTSS agar.

\section{Oscillatory dynamics of the phytoplankton community}

Our sampling targeted an anticyclonic eddy to facilitate Lagrangian sampling, and was characterized by warm, nutrient-deplete surface waters typical of the persistently oligotrophic NPSG(5,54) (Table 1). Photosynthetic picoeukaryotes, Prochlorococcus, and Crocosphaera contributed substantially to phytoplankton biomass(7) (Figure 1). POC, which includes bulk community biomass, and phytoplankton-specific biomass oscillated with significant 24-hour periodicity (Figure 1). Cell abundances and total biomass of Prochlorococcus and Crocosphaera populations increased between the first and second sampling periods (Table 1). Wind speed also increased between the first and second sampling periods, resulting in an increase in the mixed layer depth from $21 \pm 5$ to $36 \pm 6 \mathrm{~m}$. Additionally, we observed a decrease in the number of significantly diel metabolite oscillations during the second sampling interval, from 55 to 9 (Table S3). This change was likely related to the deepening of the mixed layer; however, we have insufficient evidence to investigate this hypothesis further. We therefore focus on data collected

A total of 79 targeted metabolites were detected across samples (Table S3). Total particulate metabolite concentration increased during the day and decreased at night, regardless of whether normalized to POC or PN (Figure 2). The most abundant compounds were osmolytes, 
204 like glycine betaine (GBT), homarine, DHPS, and DMSP; nucleobases (particularly guanine);

205 and amino acids related to nitrogen metabolism, such as glutamic acid and glutamine (Table S3,

206 Figure 2). At dusk, quantified metabolites totaled $1.7 \pm 0.2 \%$ of POC and $3.1 \pm 0.6 \%$ of

207 particulate nitrogen (Figure 2), with free nucleobases and amino acids representing substantial

208 pools of total cellular nitrogen (Table S4).

209 Multivariate analyses were used to determine if time of day influenced the community

210 metabolome. NMDS analysis shows that samples collected at different times were significantly

211 different. Samples collected near sunrise (6:00) were more similar to one another than those

212 collected at other times of day and are most dissimilar to samples collected near sunset

213 (ANOSIM, $R=0.19, p=0.001$, Figure S1, Table S5).

Metabolite diel periodicity

To determine whether metabolite oscillations were driven by changes in biomass or by

217 changing cell physiology resulting in changes in the intracellular concentration, we calculated

218 concentrations relative to water volume filtered, resulting in values proportional to molar

219 concentration (nmol L-1), and to POC, resulting in values proportional to nmol per $\mu$ mol POC.

220 Bulk and individual metabolite concentrations oscillated with respect to both normalizations

221 (Figure 2, Figure 3A). The molar concentration $\left(\mathrm{nmol} \mathrm{L} \mathrm{L}^{-1}\right)$ of 55 metabolites $(70 \%)$ had

222 significant 24-hour oscillations, with 26 reaching a maxima in concentration within two hours of

223 18:00 and 20 reaching their peak concentration within 2 hours of 14:00 (Figure 3, Table S3).

224 When normalized to POC (nmol $\mu \mathrm{mol} \mathrm{POC}^{-1}$ ), 37 compounds (47\%) showed diel oscillations

225 (Table S3), and the mean time of peak concentration shifted to earlier in the afternoon (Figure

226 3A). POC reflects total community biomass and detritus, so to avoid assumptions of metabolite 
source, we present molar concentrations throughout except where metabolite source can be constrained to a specific phytoplankton, in which case we present metabolite concentration normalized to the cell number or biomass of the source organism.

231 all of which exceeded the 1.2- and 1.8-fold changes of POC and the sum of FCM phytoplankton

232 biomass, respectively (Figure 4). The disaccharides trehalose and sucrose displayed the most

233 robust oscillations ( $p$-value $<1 \times 10^{-13}$, Figures 4,5 ). Trehalose and sucrose are known osmolytes, 234 and nearly all other identified osmolytes (9/10) showed diel oscillations (Figures 4, 6, Table S3). 235 Glutamic acid is the only known osmolyte that did not have a significant oscillation in molar 236 concentration (Table S3).

238 The three methionine-cycle compounds detected, S-adenosyl methionine (SAM), S-adenosyl 239 homocysteine (SAH), and methionine, showed oscillations. 5'-Methylthioadenosine (MTA) is 240 produced from SAM during polyamine synthesis and had a temporal pattern that closely matched

241 SAM (Figures 3, 6), such that SAM/MTA remained relatively constant. Pantothenate (Vitamin $242 \mathrm{~B}_{5}$ ) was one of the few compounds that peaked in the morning (Figure 3). Vitamins involved in

243 redox balance, riboflavin and niacin (Vitamins $\mathrm{B}_{2}$ and $\mathrm{B}_{3}$ ) oscillated with maxima near dusk.

244 Reduced glutathione oscillated with an afternoon peak (Figure 3).

246 Connections between metabolites and transcripts

To investigate the relationships between gene expression and metabolite concentration

248 we used the KEGG database to connect metabolites with transcripts annotated as encoding 249 proteins that directly produce or degrade each metabolite. All but four of our diel metabolites 
250 related to at least one annotated prokaryotic or eukaryotic transcript (Figure S2).

251 Glucosylglycerol, ergosterol, and isethionic acid are in the KEGG database but no transcripts

252 were annotated in our dataset as directly producing or degrading them, while homarine is not

253 included in the KEGG database.

254 Although the number of transcripts associated with each metabolite is inherently biased

255 by the databases used and the depth of sequencing, transcripts provide insight into the number

256 and identity of organisms and pathways that may be responsible for the metabolite's synthesis

257 and degradation. The orders containing Crocosphaera, Prochlorococcus, Pelagibacter ubique,

258 and other unclassified alphaproteobacteria comprised $\sim 50 \%$ of all prokaryotic transcripts that

259 could be linked to metabolites (Table S6).. Dinoflagellates (Dinophyceae), non-diatom

260 stramenopiles (Stramenopiles), haptophytes (Haptophyceae), non-metazoa opistokonts

261 (Opisthokonta), and diatoms (Bacillariophyta) comprised $\sim 70 \%$ of eukaryotic transcripts linked

262 with metabolites (Table S7). Adenosine monophosphate (AMP), SAM, and SAH stand out as the

263 diel metabolites with the largest number of associated diel transcripts, with 181, 124, and 113

264 transcripts respectively (Figure 6, Figure S2). Most diel SAM and SAH transcripts were

265 methyltransferases that convert SAM into SAH (Tables S6, S7). In most other cases, there were

266 few diel transcripts associated with a metabolite (e.g. only 6 diel genes were associated with

267 trehalose, Figure 6, Figure S2).

268 To investigate the temporal relationship between gene expression and metabolite

269 concentration, we estimated the lag-time between metabolites and transcripts that exhibited

270 significant diel periodicity. This analysis showed a broad distribution in the lag-times between 
271 metabolites and transcripts, with no predictable lag for prokaryotic or eukaryotic transcripts and

272 their associated metabolites. (Figure S3).

273

274 Disaccharide osmolytes can be attributed to cyanobacteria

275 We observed trehalose-related transcripts from eukaryotic phytoplankton and

276 Crocosphaera (Figure 6). Using published Ostreococcus cellular trehalose concentrations(20)

277 and picoeukaryote cell counts, we estimated that picoeukaryote contribution to trehalose was

$278 \quad 0.2-3.0 \mathrm{pmol} \mathrm{L}^{-1}$, a small fraction of environmental trehalose (up to $627 \mathrm{pmol} \mathrm{L}^{-1}$ ). The

279 abundance of Crocosphaera (Table 1, Figure 1) and diel oscillations in the Crocosphaera

280 transcript for trehalose 6-phosphate synthase/phosphatase (Figure 6, Table S6) suggest

281 Crocosphaera as the main contributor of trehalose during this field study. To test this hypothesis, 282 we grew Crocosphaera watsonii WH8501 under a 12:12 light:dark cycle and measured 0.8 and $2830.07 \mathrm{fmol} \mathrm{trehalose} \mathrm{cell}^{-1}$ at the end of the light and dark periods, respectively (Figure 5, Figure 284 S4). Given the Crocosphaera abundance during our sampling and assuming similar intracellular 285 concentration, this accounts for 1.8-670 pM particulate trehalose, comparable to total particulate 286 trehalose during our sampling (2.8-627 pmol L-1 across both sampling periods, Figure 5).

287 Multiple taxa expressed transcripts related to production and degradation of sucrose, 288 including Prochlorococcus (Figure 6). To assess the potential contribution of Prochlorococcus to 289 environmental sucrose concentrations, we measured the cellular sucrose quota in a culture of 290 Prochlorococcus MIT1314 harvested midday during exponential growth. Using the cellular 291 quota of sucrose in these cultures (range in biological triplicates: 1.4-2.1 amol cell ${ }^{-1}$ ) and the 
292 abundance of Prochlorococcus at the time of sampling, it is possible that all the observed sucrose

293 could have been in Prochlorococcus during this study (Figure 5).

294

295 Discussion

As a whole, the metabolites we measured comprise up to $2 \%$ of POC and $3 \%$ of PN in

297 our samples (Figure $2 \mathrm{~B}, \mathrm{C}$ ). This is a reasonable value given $\sim 80 \%$ of surface POC is comprised

298 of lipid, carbohydrate, and protein macromolecules(16,17), and DNA, RNA, and pigments

299 contribute several percent of the dry weight of actively growing microalgae(55). Metabolite

300 pools are dynamic, and an increase in the concentration of a given metabolite suggests that

301 sources of that compound (biosynthesis, uptake from dissolved pools, or polymer disassembly)

302 are greater than sinks (exudation, loss due to cell death, intracellular degradation, or polymer

303 assembly). The prevalence and amplitude of diel oscillations in metabolite concentrations reflect

304 that many members of the surface microbial community near Station ALOHA were

305 synchronized to diel light periodicity.

306

307 Community synchrony is driven by diel partitioning of anabolism, catabolism, and redox

308 maintenance

310 alternation of carbon fixation, anabolism, and growth during daylight hours and respiration,

311 catabolism, and mortality during the night (Figure 1)(2,3,5). The community metabolome

312 reflects these patterns with an overall increase in concentration throughout the day and a

313 consistent morning phenotype (Figure 2, Figure S1A,B), reflecting nighttime use of energy

314 stores and recovery from daytime oxidative stress(56). Nearly half of the diel metabolites (26/55) 
315 had peak molar concentrations near dusk (Figure 3), corresponding with a peak in carbon biomass. However, for most (46/55) diel metabolites, the daily enrichment of a metabolite exceeded that of POC or total FCM-resolvable phytoplankton biomass, which had daily fold

318 changes of 1.2 and 1.8, respectively (Figure 4). This suggests these metabolites likely had oscillations in intracellular concentration, as previously observed for many primary metabolites in non-marine cyanobacteria(57). community scale. SAM, SAH, and AMP are compounds involved in biosynthesis that had diel oscillations with daytime increases (Figures 3,6). Individual transcripts associated with these molecules had diel patterns that peaked at all times of day, across a myriad of pathways and microbial taxa (Figure 6, Figure S2). Despite this diversity in use, the sum of community activity was reflected in diel oscillations of metabolite concentrations, which were synchronized with daytime biomass accumulation. Further evidence of this daytime community-scale anabolism is the diel oscillation of pantothenate (Vitamin $\mathrm{B}_{5}$ ), a component of Coenzyme A as well as Acyl Carrier Protein. Pantothenate peaked in the morning (Figure 3), suggesting that the community was poised to assemble these cofactors for daytime biosynthesis. converted to SAH, which is then regenerated back to SAM via methionine. In addition to its role in methylation, SAM is essential for polyamine synthesis and is the most common riboswitch

334 effector in prokaryotes(58). SAM riboswitches have been observed in native Station ALOHA

336 ratio was at a minimum during the day (Figure 6). This ratio reflects methylation potential,

337 suggesting that the demand for methylation outstripped the supply of SAM in the light. Over the 
dark period, SAM/SAH ratios recovered, suggesting that catabolic processes dominated and the need for SAM was diminished. Many cells require cobalamin to catalyze the reactions that

340 regenerate methionine, and SAH is elevated relative to SAM during cobalamin stress as cells

341 struggle to complete the cycle(25). Thus, it is possible that the lower SAM/SAH ratio

342 additionally reflects a daytime increase in cobalamin usage.

344 produced during photosynthesis accumulate over the day and present a continuing challenge for

345 cells at night(60). Riboflavin and niacin (vitamins $\mathrm{B}_{2}$ and $\mathrm{B}_{3}$ ) are involved in redox balance and 346 show similar daytime accumulations (Figure 3, Table S3). These are precursors to FMN/FAD

347 and NAD/NADP, respectively, and reflect community-wide diel patterns of redox processes.

348 Cyanobacteria manage excess energy during the day by storing glycogen and producing small

349 molecules that can either be stored or excreted(56,60-63). At night, glycogen is catabolized via

350 hydrolysis followed by glycolysis or the oxidative pentose phosphate pathway (OPPP),

351 producing the reductant sources NADH and NADPH. Gluconic acid accumulation during the

352 day (Figures 3, 4) may reflect less flux through OPPP during the day, while photosynthesis

353 produces NADPH, followed by a switch towards OPPP at night(56). Reduced glutathione also

354 showed a daytime peak (Figure 3), as has been observed in cultures and field studies(64),

355 possibly reflecting production to compensate for increased oxidative stress in the day, and a

356 subsequent decrease in production and oxidation of the residual pool overnight. 
361 this observation was glutamic acid, which plays a critical role in regulating nitrogen assimilation

362 in addition to its osmotic properties(65). In the absence of fluctuations in salinity or temperature,

363 oscillations in osmolyte concentrations occurred in excess of or out of sync with biomass

364 oscillations and point to alternative roles for this compound group(65) (Figure 4, Table S3).

365 Intracellular accumulation of osmolytes occurred predominantly during the day when electron

366 flow through the photosystems and the Calvin Cycle exceeds that required to maintain maximum

367 division rates. The resulting need to dissipate reductant is typically channeled into the production

368 of carbohydrates like glycogen $(8,56,63)$, exopolymeric substances $(70,71)$, or into storage

369 lipids(12,72). These energy stores are used to fuel cellular respiration and other activities at

370 night, such as protein synthesis and preparing cells for photosynthesis $(12,56,63,72)$. Unlike

371 starch and storage lipids, osmolytes do not necessarily need to go through hydrolysis, $\beta$ -

372 oxidation, or glycolysis prior to entering the TCA cycle, and could be used as readily available

373 substrates for energy production and as biosynthetic intermediates while macromolecular pools

374 are being mobilized by the cell(61).

375 Trehalose was the most prominent diurnally oscillating compound (Figures 4, 5).

376 Trehalose is an osmolyte produced by the unicellular diazotroph Crocosphaera $(68,73)$, some

377 heterotrophic bacteria, and some phytoplanktonic picoeukaryotes, including Ostreococcus(20).

378 Transcriptomic evidence motivated us to measure trehalose in cultures of Crocosphaera, which

379 revealed differences in intracellular trehalose at the beginning and end of the day. Assuming

380 trehalose in the environment is produced primarily by Crocosphaera, our results strongly suggest

381 that intracellular trehalose concentrations have diel oscillations in the field (Figure 5).

$382 \quad$ Crocosphaera temporally separate photosynthesis and nitrogen fixation to protect

383 nitrogenase from oxygen(74-76), they therefore need energy at night to draw down cellular 
384 oxygen and fuel nitrogen fixation $(77,78)$. Crocosphaera has at least one gene encoding a protein

385 homologous to glycoside hydrolases, family 15(79), which contains enzymes that hydrolyze a

386 variety of glycosidic bonds, including trehalose. Thus, it is possible that Crocosphaera use

387 trehalose as a fuel for generating the electrons and ATP required for nitrogen fixation. Using the

388 stoichiometry of these reactions $(77,80)$, we estimated that trehalose catabolism could have fueled

$3899-28 \%$ of the nighttime nitrogen fixation during this expedition(7) (calculation in supplemental

390 material). As much as $60 \%$ of total dark respiration by Crocosphaera is used to draw down

391 cellular oxygen rather than to directly fuel nitrogen fixation(77), and, if we adjust our calculation

392 accordingly, trehalose can produce 3.6-11\% of the required respiratory substrates needed for

393 Crocosphaera to effectively fix nitrogen at the rates measured(7).

394 The flux of carbon through trehalose may be an indicator of the accumulation and

395 degradation of a larger glycogen pool that accumulates during the day and is used at night(81).

396 Shi et al. (2010) suggest that Crocosphaera cells are depleted of storage compounds at night,

397 since prolonged dark does not result in increased nitrogen fixation(82). If this hypothesis is

398 correct, the total amount of nitrogen fixation possible is limited by the amount of energy stored

399 in substrates such as trehalose and glycogen during daytime, and the ability to accumulate and

400 use these compounds could have impacts on the nitrogen budget of the microbial community.

$401 \quad$ Another disaccharide osmolyte, sucrose, displayed an oscillation with a maximum daily

402 concentration at 22:00. Sucrose is the major compatible solute in high-light Prochlorococcus

403 (67), and the observed environmental variation may reflect the in situ accumulation and use of

404 glycogen by Prochlorococcus. Though other organisms also expressed sucrose related genes

405 (Figure 6), Prochlorococcus was the numerically dominant sucrose-producing organism detected

406 in these populations (Table 1) and is known to accumulate polysaccharides during the day, 
407 particularly under nitrogen limitation(83). If we assume that cellular quotas of sucrose in

408 Prochlorococcus grown in culture are like those in the environment, Prochlorococcus alone

409 could explain the sucrose concentrations seen in the environment (Figure 5). Sucrose had a diel

410 oscillation when normalized to Prochlorococcus cell counts and biomass (Figure 5, Figure S5).

411 These potential intracellular oscillations lead us to hypothesize that Prochlorococcus uses

412 sucrose for energy storage and not only as a compatible solute, as has been observed in non-

413 marine cyanobacteria $(57,61)$.

Homarine and DMSP are known eukaryotic osmolytes $(65,66,69,84)$. Here the amplitude

415 and timing of the diel oscillations in these two compounds differ from those observed in

416 phytoplankton picoeukaryote biomass (Figure 4), suggesting that these compatible solutes play

417 additional roles within the microbial community. This diversity of functions is well established

418 for DMSP, which influences grazing behaviors and can function as an antioxidant(69,85). DMSP

419 is also a carbon and reduced sulfur source in the microbial community, with uptake and

420 assimilation both tied to light availability $(86,87)$. In our analysis, the only transcript related to

421 DMSP encodes a SAR11 DMSP demethylase required for DMSP degradation (Figure 6). A

422 dearth of data on the roles of homarine in marine microbes and a lack of genetic information

423 about homarine synthesis and degradation limit our ability to infer the sources and sinks for this

424 abundant compound. The high concentration and diel dynamics of homarine calls for further

425 investigation.

426 Both isethionic acid and DHPS are associated with fast growing eukaryotes that need to

427 mobilize cellular machinery to transport materials into the mitochondria for respiration $(27,88)$,

428 and recent work has suggested that DHPS has potential osmotic capabilities(27). These two

429 metabolites had large diel oscillations implicating them as temporary stores of energy or 
430 intermediates that can be mobilized quickly. Our data implicates SAR11 and Rhodobacteraceae 431 as likely DHPS degraders at Station ALOHA (Figure 6), although genes for the production of

432 DHPS are not in the KEGG database and thus were not identified by our analyses. If production

433 and degradation of these compounds are separated along phylogenic lines(31) then these

434 compounds are likely excreted into the dissolved phase by eukaryotes and subsequently available

435 for use by bacteria, as suggested in Durham et al. (2019). This may explain the midday maximal 436 expression of a $h p s N$-like Rhodobacteraceae DHPS degradation gene (Figure 6).

442 oscillations observed in this study call for further investigation into the hypothesis that these

443 compounds are important substrates for community interactions and resources for the microbial

444 loop. For compounds that exhibited diel oscillations, the difference between the daily maximum 445 and minimum values provides a daily net production and degradation rate. We estimated a total 446 net turnover rate of over $27 \mathrm{nmol} \mathrm{C} \mathrm{L}^{-1} \mathrm{~d}^{-1}$ from our targeted metabolites, with several 447 metabolites exhibiting individual turnover rates of over $1 \mathrm{nmol} \mathrm{C} \mathrm{L}{ }^{-1} \mathrm{~d}^{-1}$, including arachidonic 448 acid, trehalose, homarine, sucrose, GBT, glucosylglycerol, and DHPS (Table S3). These are

449 conservative estimates since the instantaneous flux may be much higher than the daily net 450 change and we did not measure excretion of metabolites into the dissolved pool. For example,

451 DMSP has a turnover time of 4.5 hours at Station ALOHA(86) and has been shown to be 452 produced at night and during the day(93), both observations would substantially increase the 
453 baseline estimate of DMSP production which does not account for rapid turnover and only

454 includes a daytime increase in concentration. While the fate of the metabolites measured here

455 remain unclear, conservative estimates of carbon and nitrogen flux through these small pools was

456 large, comprising around $2 \%$ of the ${ }^{14} \mathrm{C}$ based estimates of primary productivity during this

457 study(12). These compounds are potentially used for cellular requirements by the organisms

458 synthesizing them, as discussed above, or released into the labile dissolved pool. When they

459 enter the dissolved pool through excretion or cell lysis, these compounds are important

460 components of the labile dissolved organic matter pool(91) and play a role in organism

461 interactions $(94,95)$.

462

463 Conclusions

The light-dark cycle plays a dominant role in structuring marine microbial activity.

465 Previous work has shown diel oscillations of community processes, such as daily accumulation

466 and depletion of POC(2), and diel oscillations of transcriptional activity, which have provided

467 new information on temporal dynamics and raise hypotheses about the activity of individual

468 taxa $(9,10)$. Measurements of in situ metabolites in native planktonic microbial populations

469 reported here support the hypotheses that diverse microbial taxa in the NPSG are synchronized to

470 daily oscillations of light energy and photosynthesis, with metabolites accumulated during the

471 day and depleted at night. The diel synchrony of ubiquitously used primary metabolites shows

472 the extent to which photoautotrophic organisms dominate the community and drive anabolic

473 processes during the day and catabolic processes at night. The combination of transcript

474 abundances, metabolite concentrations, and taxa-specific biomass in the field and in culture

475 allows us to postulate that Crocosphaera uses trehalose as a short-term energy source to drive 
476 nighttime nitrogen fixation. Trehalose and the other osmolytes we measured are highly abundant

477 in cells and, in addition to playing multiple roles in their producers, likely fuel the metabolism of

478 heterotrophic bacteria. Metabolite concentrations cannot be predicted from transcripts in a single

479 organism in pure culture, let alone in a complex natural community. Pairing quantitative

480 measurements of particulate metabolites with transcriptomes is a key step toward understanding

481 how regularly oscillating gene expression in microbial communities is reflected in the net

482 community processes we observe and further elucidates the currencies of the microbial

483 community.

\section{Acknowledgements}

485 The authors acknowledge A. Hynes, N. Kellogg, R. Lionheart, M. Motukuri, and A. Wied for

486 assistance with lab and data analysis; J.S. Weitz and D. Muratore for productive discussions and

487 feedback; A. E. White for the POC and PN data; J.P. Zehr and M. Hogan for providing

488 Crocosphaera WH8501; A. Coe and S.W. Chisholm for providing Prochlorococcus MIT1314;

489 the crew and scientific party of the R/V Kilo Moana during HOE-Legacy 2A. This work was

490 supported by grants from the Simons Foundation (LS Award ID: 385428, A.E.I.; SCOPE Award

491 ID 329108, A.E.I., E.F.D., E.V.A.; SCOPE Award ID 426570, E.V.A.; Award ID 598819,

492 K.R.H.), the National Science Foundation (NSF OCE-1228770 and OCE-1205232 to A.E.I.,

493 NSF OCE-160019 to R.D.G, NSF GRFP to A.K.B. and K.R.H., NSF IGERT Program on Ocean

494 Change to A.K.B), and the Gordon and Betty Moore Foundation (Grant \#3777 to E.F.D.)

495 Competing interests:

496 The authors declare no conflict of interest.

497 Data availability

498 Information for the KM1513/HOE Legacy II cruise can be found online at

499 http:/hahana.soest.hawaii.edu/hoelegacy/hoelegacy.html. Raw sequence data for the diel 
500 eukaryotic metatranscriptomes are available in the NCBI Sequence Read Archive under

501 BioProject ID PRJNA492142. Raw sequence data for the prokaryotic metatranscriptomes are

502 available in the NCBI Sequence Read Archive under BioProject ID PRJNA358725. Raw and

503 processed metabolomics data are available in Metabolomics Workbench under Project ID

504 PR000926, currently embargoed until July 1, 2020 but available upon publication.

505

506 Author contributions

507 AEI, EVA, and EFD designed the study. AKB, LTC, FOA, BPD, and FR collected the samples.

$508 \mathrm{AKB}, \mathrm{LTC}$, and KRH performed the metabolite sample processing and analysis. FOA, RDG, and 509 BPD performed the metatranscriptomic sample processing and analysis. FR performed the flow 510 cytometry sample processing and analyses. AKB, KRH, and AEI contributed to data

511 interpretation and visualization. AKB and AEI drafted the paper and incorporated revisions from 512 all authors. 


\section{References}

1. Field CB, Behrenfeld MJ, Randerson JT, Falkowski P. Primary production of the biosphere: integrating terrestrial and oceanic components. Science. 1998;281(5374):23740.

2. White AE, Barone B, Letelier RM, Karl DM. Productivity diagnosed from the diel cycle of particulate carbon in the North Pacific Subtropical Gyre. Geophys Res Lett. 2017;44(8):3752-60.

3. Ribalet F, Swalwell J, Clayton S, Jiménez V, Sudek S, Lin Y, et al. Light-driven synchrony of Prochlorococcus growth and mortality in the subtropical Pacific gyre. Proc Natl Acad Sci U S A. 2015;1424279112-.

4. Ferrón S, Wilson ST, Martínez-garcía S, Quay PD, Karl DM. Metabolic balance in the mixed layer of the oligotrophic North Pacific Ocean from diel changes in O2/Ar saturation ratios. Geophys Res Lett. 2015;3421-30.

5. Hu SK, Connell PE, Mesrop LY, Caron DA. A Hard Day’s night: Diel shifts in microbial eukaryotic activity in the North Pacific Subtropical Gyre. Front Mar Sci. 2018;5(OCT):351.

6. Aylward FO, Boeuf D, Mende DR, Wood-Charlson EM, Vislova A, Eppley JM, et al. Diel cycling and long-term persistence of viruses in the ocean's euphotic zone. Proc Natl Acad Sci U S A. 2017;114(43):11446-51.

7. Wilson ST, Aylward FO, Ribalet F, Barone B, Casey JR, Connell PE, et al. Coordinated regulation of growth, activity and transcription in natural populations of the unicellular nitrogen-fixing cyanobacterium Crocosphaera. Nat Microbiol. 2017;2(July):1-20.

8. Halsey KH, Jones BM. Phytoplankton Strategies for Photosynthetic Energy Allocation. Ann Rev Mar Sci. 2015;7(1):265-97.

9. Aylward FO, Eppley JM, Smith JM, Chavez FP, Scholin C a., DeLong EF. Microbial community transcriptional networks are conserved in three domains at ocean basin scales. Proc Natl Acad Sci. 2015;112(17):201502883.

10. Ottesen E a., Young CR, Gifford SM, Eppley JM, Marin R, Schuster SC, et al. Multispecies diel transcriptional oscillations in open ocean heterotrophic bacterial assemblages. Science. (80- ). 2014;345(6193):207-12.

11. Waldbauer JR, Rodrigue S, Coleman ML, Chisholm SW, Sun Z. Transcriptome and Proteome Dynamics of a Light-Dark Synchronized Bacterial Cell Cycle. Lin S, editor. PLoS One. 2012;7(8):e43432.

12. Becker KW, Collins JR, Durham BP, Groussman RD, White AE, Fredricks HF, et al. Daily changes in phytoplankton lipidomes reveal mechanisms of energy storage in the open ocean. Nat Commun. 2018;9(1):5179.

13. Foy RH, Smith R V. The role of carbohydrate accumulation in the growth of planktonic oscillatoria species. Br Phycol J. 1980;15(2):139-50.

14. Muratore D, Boysen AK, Harke MJ, Becker KW, Casey JR, Coesel SN, et al. Community-scale Synchronization and Temporal Partitioning of Gene Expression, Metabolism and Lipids in Oligotrophic Ocean Surface Waters. bioRxiv. 2020;

15. Becker KW, Harke MJ, Mende DR, Muratore D, Weitz JS, Delong EF, et al. Combined pigment and metatranscriptomic analysis reveals highly synchronized diel patterns of phenotypic light response across domains in the open oligotrophic ocean. bioRxiv. 2020;

16. Wakeham SG, Lee C, Hedges JI, Hernes PJ, Peterson MJ. Molecular indicators of diagenetic status in marine organic matter. Geochim Cosmochim Acta. 
602

603

604

605

1997;61(24):5363-9.

17. Hedges JI, Baldock JA, Gélinas Y, Lee C, Peterson ML, Wakeham SG. The biochemical and elemental compositions of marine plankton: A NMR perspective. Mar Chem. 2002;78(1):47-63.

18. Moran MA. The global ocean microbiome. Science (80- ). 2015;350(6266):aac8455aac8455.

19. Boysen AK, Heal KR, Carlson LT, Ingalls AE. Best-Matched Internal Standard Normalization in Liquid Chromatography-Mass Spectrometry Metabolomics Applied to Environmental Samples. Anal Chem. 2018;90(2).

20. Hirth M, Liverani S, Mahlow S, Bouget FY, Pohnert G, Sasso S. Metabolic profiling identifies trehalose as an abundant and diurnally fluctuating metabolite in the microalga Ostreococcus tauri. Metabolomics. 2017;13(6):68.

21. Kujawinski EB, Longnecker K, Alexander H, Dyhrman ST, Fiore CL, Haley ST, et al. Phosphorus availability regulates intracellular nucleotides in marine eukaryotic phytoplankton. Limnol Oceanogr Lett. 2017;2(4):119-29.

22. Heal KR, Qin W, Ribalet F, Bertagnolli AD, Coyote-Maestas W, Hmelo LR, et al. Two distinct pools of B12 analogs reveal community interdependencies in the ocean. Proc Natl Acad Sci U S A. 2016;201608462.

23. Llewellyn CA, Sommer U, Dupont CL, Allen AE, Viant MR. Using community metabolomics as a new approach to discriminate marine microbial particulate organic matter in the western English Channel. Prog Oceanogr. 2015;137:421-33.

24. Johnson WM, Longnecker K, Kido Soule MC, Arnold WA, Bhatia MP, Hallam SJ, et al. Metabolite composition of sinking particles differs from surface suspended particles across a latitudinal transect in the South Atlantic. Limnol Oceanogr. 2019;65(1):111-27.

25. Heal KR, Kellogg NA, Carlson LT, Lionheart RM, Ingalls AE. Metabolic Consequences of Cobalamin Scarcity in the Diatom Thalassiosira pseudonana as Revealed Through Metabolomics. Protist. 2019;170(3):328-48.

26. Torstensson A, Young JN, Carlson LT, Ingalls AE, Deming JW. Use of exogenous glycine betaine and its precursor choline as osmoprotectants in Antarctic sea-ice diatoms. J Phycol. 2019;

27. Durham BP, Boysen AK, Carlson LT, Groussman RD, Heal KR, Cain KR, et al. Sulfonate-based networks between eukaryotic phytoplankton and heterotrophic bacteria in the surface ocean. Nat Microbiol. 2019;4(10):1706-15.

28. Bundy JG, Davey MP, Viant MR. Environmental metabolomics: a critical review and future perspectives. Metabolomics. 2009;5(1):3-21.

29. Goulitquer S, Potin P, Tonon T. Mass spectrometry-based metabolomics to elucidate functions in marine organisms and ecosystems. [Internet]. Vol. 10, Marine drugs. Molecular Diversity Preservation International; 2012. 849-80 p.

30. Amin S a., Hmelo LR, Tol HM van, Durham BP, Carlson LT, Heal KR, et al. Interaction and signalling between a cosmopolitan phytoplankton and associated bacteria. Nature. 2015;522(7554):98-101.

31. Durham BP, Sharma S, Luo H, Smith CB, Amin SA, Bender SJ, et al. Cryptic carbon and sulfur cycling between surface ocean plankton. Proc Natl Acad Sci U S A. 2014;in review(2):453-7.

32. Moran MA, Kujawinski EB, Stubbins A, Fatland R, Aluwihare LI, Buchan A, et al. Deciphering ocean carbon in a changing world. Proc Natl Acad Sci U S A. 


\section{6;1514645113-.}

33. Swalwell JE, Ribalet F, Armbrust EV. SeaFlow: A novel underway flow-cytometer for continuous observations of phytoplankton in the ocean. Limnol Oceanogr Methods. 2011;9(10):466-77.

34. Ribalet F, Berthiaume C, Hynes A, Swalwell J, Carlson M, Clayton S, et al. SeaFlow data v1, high-resolution abundance, size and biomass of small phytoplankton in the North Pacific. Sci Data. 2019;6(1):277.

35. Bligh EG, Dyer WJ. A Rapid Method Of Total Lipid Extraction And Purification. Can J Biochem Physiol. 2010;37(1):911-7.

36. MacLean B, Tomazela DM, Shulman N, Chambers M, Finney GL, Frewen B, et al. Skyline: an open source document editor for creating and analyzing targeted proteomics experiments. Bioinformatics. 2010;26(7):966-8.

37. Spielmeyer A, Pohnert G. Direct quantification of dimethylsulfoniopropionate (DMSP) with hydrophilic interaction liquid chromatography/mass spectrometry. J Chromatogr B Anal Technol Biomed Life Sci. 2010;878(31):3238-42.

38. Mende DR, Bryant JA, Aylward FO, Eppley JM, Nielsen T, Karl DM, et al. Environmental drivers of a microbial genomic transition zone in the ocean's interior. Nat Microbiol. 2017;2(10):1367-73.

39. Kiełbasa SM, Wan R, Sato K, Horton P, Frith MC. Adaptive seeds tame genomic sequence comparison. Genome Res. 2011;21(3):487-93.

40. Gifford SM, Becker JW, Sosa OA, Repeta DJ, DeLong EF. Quantitative Transcriptomics Reveals the Growth- and Nutrient-Dependent Response of a Streamlined Marine Methylotroph to Methanol and Naturally Occurring Dissolved Organic Matter. MBio. 2016;7(6):e01279-16.

41. Kanehisa M, Goto S. KEGG: Kyoto Encyclopedia of Genes and Genomes. Nucleic Acids Res. 2000;28(1):27-30.

42. Grabherr MG, Haas BJ, Yassour M, Levin JZ, Thompson DA, Amit I, et al. Full-length transcriptome assembly from RNA-Seq data without a reference genome. Nat Biotechnol. 2011;29(7):644-52.

43. Buchfink B, Xie C, Huson DH. Fast and sensitive protein alignment using DIAMOND. Nat Methods. 2015;12(1):59-60.

44. Eddy SR. Accelerated profile HMM searches. Pearson WR, editor. PLoS Comput Biol. 2011;7(10):e1002195.

45. Aramaki T, Blanc-Mathieu R, Endo H, Ohkubo K, Kanehisa M, Goto S, et al. KofamKOALA: KEGG ortholog assignment based on profile HMM and adaptive score threshold. Valencia A, editor. Bioinformatics. 2019;36(7):2251-2.

46. Bray NL, Pimentel H, Melsted P, Pachter L. Near-optimal probabilistic RNA-seq quantification. Nat Biotechnol. 2016;34(5):525-7.

47. Tenenbaum D. KEGGREST: Client-side REST access to KEGG. R package; 2017.

48. Thaben PF, Westermark PO. Detecting rhythms in time series with RAIN. J Biol Rhythms. 2014;29(6):391-400.

49. Benjamini Y, Hochberg Y. Controlling the False Discovery Rate: A Practical and Powerful Approach to Multiple Testing. J R Stat Soc Ser B. 1995;57(1):289-300.

50. Chen YB, Zehr JP, Mellon M. Growth and nitrogen fixation of the diazotrophic filamentous nonheterocystous cyanobacterium Trichodesmium sp. IMS 101 in defined media: Evidence for a circadian rhythm. J Phycol. 1996;32(6):916-23. 
652 51. Becker JW, Hogle SL, Rosendo K, Chisholm SW. Co-culture and biogeography of

653

654

655

656

657

658

659

660

661

662

663

664

665

666

667

668

669

670

671

672

673

674

675

676

677

678

679

680

681

682

683

684

685

686

687

688

689

690

691

692

693

694

695

696

697

Prochlorococcus and SAR11. ISME J. 2019;13(6):1506-19.

52. Moore LR, Coe A, Zinser ER, Saito MA, Sullivan MB, Lindell D, et al. Culturing the marine cyanobacterium Prochlorococcus. Limnol Oceanogr Methods. 2007;5(10):353-62.

53. Jeffrey Morris J, Zinser ER. Continuous hydrogen peroxide production by organic buffers in phytoplankton culture media. Post A, editor. J Phycol. 2013;49(6):1223-8.

54. Karl DM, Church MJ. Microbial oceanography and the Hawaii Ocean Time-series programme. Nat Rev Microbiol. 2014;12(10):699-713.

55. Finkel Z V., Follows MJ, Liefer JD, Brown CM, Benner I, Irwin AJ. Phylogenetic Diversity in the Macromolecular Composition of Microalgae. Humbert J-F, editor. PLoS One. 2016;11(5):e0155977.

56. Welkie DG, Rubin BE, Diamond S, Hood RD, Savage DF, Golden SS. A Hard Day's Night: Cyanobacteria in Diel Cycles. Trends Microbiol. 2018;27(3):231-42.

57. Will SE, Henke P, Boedeker C, Huang S, Brinkmann H, Rohde M, et al. Day and night: Metabolic profiles and evolutionary relationships of six axenic non-marine cyanobacteria. Katz LA, editor. Genome Biol Evol. 2019;11(1):270-94.

58. Winkler WC, Nahvi A, Sudarsan N, Barrick JE, Breaker RR. An mRNA structure that controls gene expression by binding S-adenosylmethionine. Nat Struct Biol.

2003;10(9):701-7.

59. Shi Y, Tyson GW, DeLong EF. Metatranscriptomics reveals unique microbial small RNAs in the ocean's water column. Nature. 2009;459(7244):266-9.

60. Latifi A, Ruiz M, Zhang CC. Oxidative stress in cyanobacteria [Internet]. Vol. 33, FEMS Microbiology Reviews. Narnia; 2009. p. 258-78.

61. Halsey K, Milligan A, Behrenfeld M. Contrasting Strategies of Photosynthetic Energy Utilization Drive Lifestyle Strategies in Ecologically Important Picoeukaryotes.

Metabolites. 2014;4(2):260-80.

62. Bertilsson S, Berglund O, Pullin M, Chisholm S. Release of dissolved organic matter by Prochlorococcus. Vol. 55, Vie Et Milieu. 2005. p. 225-31.

63. Shinde S, Zhang X, Singapuri SP, Kalra I, Liu X, Morgan-Kiss RM, et al. Glycogen Metabolism Supports Photosynthesis Start through the Oxidative Pentose Phosphate Pathway in Cyanobacteria. Plant Physiol. 2020;182(1):507-17.

64. Dupont CL, Goepfert TJ, Lo P, Wei L, Ahner BA. Diurnal cycling of glutathione in marine phytoplankton: Field and culture studies. Limnol Oceanogr. 2004;49(4):991-6.

65. Welsh DT. Ecological significance of compatible solute accumulation by microorganisms: from single cells to global climate. FEMS Microbiol Rev. 2000;24(3):263-90.

66. Gebser B, Pohnert G. Synchronized regulation of different zwitterionic metabolites in the osmoadaption of phytoplankton. Mar Drugs. 2013;11(6):2168-82.

67. Klähn S, Steglich C, Hess WR, Hagemann M. Glucosylglycerate: A secondary compatible solute common to marine cyanobacteria from nitrogen-poor environments. Environ Microbiol. 2010;12(1):83-94.

68. Klähn S, Hagemann M. Compatible solute biosynthesis in cyanobacteria. Environ Microbiol. 2011;13(3):551-62.

69. Yancey PH. Organic osmolytes as compatible, metabolic and counteracting cytoprotectants in high osmolarity and other stresses. J Exp Biol. 2005;208(15):2819-30.

70. DiTullio G, Laws E. Diel periodicity of nitrogen and carbon assimilation in five species of marine phytoplankton: accuracy of methodology for predicting $\mathrm{N}$-assimilation rates and 
N/C composition ratios. Mar Ecol Prog Ser. 1986;32:123-32.

71. Mühlenbruch M, Grossart HP, Eigemann F, Voss M. Mini-review: Phytoplankton-derived polysaccharides in the marine environment and their interactions with heterotrophic bacteria [Internet]. Vol. 20, Environmental Microbiology. Wiley/Blackwell (10.1111);

72. Lacour T, Sciandra A, Talec A, Mayzaud P, Bernard O. Diel variations of carbohydrates and neutral lipids in nitrogen-sufficient and nitrogen-starved cyclostat cultures of isochrysis sp. J Phycol. 2012;48(4):966-75.

73. Pade N, Compaoré J, Klähn S, Stal LJ, Hagemann M. The marine cyanobacterium Crocosphaera watsonii WH8501 synthesizes the compatible solute trehalose by a laterally acquired OtsAB fusion protein. Environ Microbiol. 2012;14(5):1261-71.

74. Dron A, Rabouille S, Claquin P, Le Roy B, Talec A, Sciandra A. Light-dark (12:12) cycle of carbon and nitrogen metabolism in Crocosphaera watsonii WH8501: relation to the cell cycle. Environ Microbiol. 2012;14(4):967-81.

75. Mohr W, Intermaggio MP, LaRoche J. Diel rhythm of nitrogen and carbon metabolism in the unicellular, diazotrophic cyanobacterium Crocosphaera watsonii WH8501. Environ Microbiol. 2010;12(2):412-21.

76. Mitsui A, Kumazawa S, Takahashi A, Ikemoto H, Cao S, Arai T. Strategy by which nitrogen-fixing unicellular cyanobacteria grow photoautotrophically. Nature. 1986;323(6090):720-2.

77. Großkopf T, LaRoche J. Direct and indirect costs of dinitrogen fixation in Crocosphaera watsonii WH8501 and possible implications for the nitrogen cycle. Front Microbiol. 2012;3(JUL):236.

78. Inomura K, Bragg J, Follows MJ. A quantitative analysis of the direct and indirect costs of nitrogen fixation: a model based on Azotobacter vinelandii. ISME J. 2017;11(1):166-75.

79. Bench SR, Heller P, Frank I, Arciniega M, Shilova IN, Zehr JP. Whole genome comparison of six Crocosphaera watsonii strains with differing phenotypes. J Phycol. 2013;49(4):786-801.

80. Sohm JA, Webb EA, Capone DG. Emerging patterns of marine nitrogen fixation. Nat Rev Microbiol. 2011;9(7):499-508.

81. Saito MA, Bertrand EM, Dutkiewicz S, Bulygin V V, Moran DM, Monteiro FM, et al. Iron conservation by reduction of metalloenzyme inventories in the marine diazotroph Crocosphaera watsonii. Proc Natl Acad Sci U S A. 2011;108(6):2184-9.

82. Shi T, Ilikchyan I, Rabouille S, Zehr JP. Genome-wide analysis of diel gene expression in the unicellular N(2)-fixing cyanobacterium Crocosphaera watsonii WH 8501. ISME J. 2010;4(5):621-32.

83. Szul MJ, Dearth SP, Campagna SR, Zinser ER. Carbon Fate and Flux in Prochlorococcus under Nitrogen Limitation. Gutierrez M, editor. mSystems. 2019;4(1):e00254-18.

84. Fenizia S, Thume K, Wirgenings M, Pohnert G. Ectoine from bacterial and algal origin is a compatible solute in microalgae. Mar Drugs. 2020;18(1):42.

85. Sunda W, Kieber DJ, Kiene RP, Huntsman S. An antioxidant function for DMSP and DMS in marine algae. Nature. 2002;418(6895):317-20.

86. Del Valle DA, Kiene RP, Karl DM. Effect of visible light on dimethylsulfoniopropionate assimilation and conversion to dimethylsulfide in the North Pacific Subtropical Gyre. Aquat Microb Ecol. 2012;66(1):47-62.

87. Moran MA, Durham BP. Sulfur metabolites in the pelagic ocean [Internet]. Vol. 17, 
Nature Reviews Microbiology. Nature Publishing Group; 2019. p. 665-78.

88. Boroujerdi AFB, Lee PA, DiTullio GR, Janech MG, Vied SB, Bearden DW. Identification of isethionic acid and other small molecule metabolites of Fragilariopsis cylindrus with nuclear magnetic resonance. Anal Bioanal Chem. 2012;404(3):777-84.

89. Clifford EL, Varela MM, De Corte D, Bode A, Ortiz V, Herndl GJ, et al. Taurine Is a Major Carbon and Energy Source for Marine Prokaryotes in the North Atlantic Ocean off the Iberian Peninsula. Microb Ecol. 2019;78(2):299-312.

90. Kirchman DL, Hodson RE. Metabolic regulation of amino acid uptake in marine waters. Limnol Oceanogr. 1986;31(2):339-50.

91. Poretsky RS, Sun S, Mou X, Moran MA. Transporter genes expressed by coastal bacterioplankton in response to dissolved organic carbon. Environ Microbiol. 2010;12(3):616-27.

92. Kirchman DL. The Contribution of Monomers and other Low-Molecular Weight Compounds to the Flux of Dissolved Organic Material in Aquatic Ecosystems. In: Aquatic Ecosystems. Academic Press; 2003. p. 217-41.

93. Bucciarelli E, Sunda WG, Belviso S, Sarthou G. Effect of the diel cycle on production of dimethylsulfoniopropionate in batch cultures of Emiliania huxleyi. Aquat Microb Ecol. 2007;48(1):73-81.

94. Fu H, Uchimiya M, Gore J, Moran MA. Ecological drivers of bacterial community assembly in synthetic phycospheres. Proc Natl Acad Sci U S A. 2020;117(7):3656-62.

95. Poulin RX, Lavoie S, Siegel K, Gaul DA, Weissburg MJ, Kubanek J. Chemical encoding of risk perception and predator detection among estuarine invertebrates. Proc Natl Acad Sci U S A. 2018;115(4):662-7. 


\section{Figure Captions}

769 Table 1

Wind speed and surface mixed layer physical and biological variables over the two sampling periods. Salinity, temperature, and dissolved oxygen (corrected with bottle measurements) are from the CTD between 13-17 m. N+N and heterotrophic bacteria abundance are measured from discrete samples at $15 \mathrm{~m}$. Particulate organic carbon (from underway beam attenuation), particulate nitrogen, Prochlorococcus, Synechococcus, photosynthetic picoeukaryotes, and Crocosphaera are measured from the ship-underway water intake near $7 \mathrm{~m}$.

Figure 1

Top: Hourly averages of POC from beam attenuation (black line, RAIN fdr-corrected $p<0.001$ ), total phytoplankton carbon biomass from flow cytometry (phytoplankton biomass, navy line, RAIN fdr-corrected $p<0.001$ ), and the difference between the two (grey line, RAIN fdr-corrected $p>0.05$ ). Bottom: Hourly averages of population specific carbon biomass of Prochlorococcus, Synechococcus, Crocosphaera, and photosynthetic picoeukaryotes (defined here as $2-4 \mu \mathrm{m}$ ) from flow cytometry, with shaded area representing the 95\% confidence interval RAIN fdr-corrected $p$ $<0.001$ for all four populations), note the $\log _{10}$-scaled y-axis. Breaks in the lines are due to short periods of instrument malfunction. The two sampling periods referred to in the text are indicated above the figure.

Figure 2

791

Average targeted metabolite composition at dawn (06:00) and dusk (18:00) from July $26^{\text {th }}-$ July $28^{\text {th }}$ ( $n=9$ for each time point), shown as the estimated particulate metabolite concentration (A), the percent of particulate organic carbon (B), and the percent of the particulate nitrogen (C). "Other" contains the sum of the rest of the metabolites (64 compounds). Osmolytes are in bold. Metabolites are arranged according to their average molar concentration at 6:00. Note the different y-axis scales.

\section{Figure 3}

Time of day that significantly diel compounds peak in the first sampling period (A). Surface light (photosynthetically active radiation, PAR, $\mathrm{x} 10 \mathrm{nmol}$ photon $\mathrm{m}^{-2} \mathrm{~s}^{-1}$ ) (B). Heat map showing the $z$-score standardized concentrations of POC and of metabolites (nmol L ${ }^{-1}$ ) determined to be significantly diel in the first sampling period, arranged by time of peak concentration $(\mathrm{C})$.

\section{Figure 4}

Peak time vs average daily fold change for each metabolite (circles, nmol L-1), POC from beam attenuation and phytoplankton biomass from flow cytometry (squares, $\mu \mathrm{g} \mathrm{C} \mathrm{L}^{-1}$ ). Grey color indicates the level of significance (fdr corrected $p$-value) of the 24-hour oscillation. Red outlines indicate that the compound is an osmolyte. Select compounds and all biomass estimates are labeled $($ croco $=$ Crocosphaera, synecho $=$ Synechococcus, prochloro $=$ Prochlorococcus, picoeuks $=$ photosynthetic picoeukaryotes, total phytos $=$ total phytoplankton biomass from underway flow cytometry). Dashed line is at a 2-fold change, which is above that for POC and total picophytoplankton biomass. The inset shows the distribution of fold-change in nonsignificant compounds. These compounds have variability even though they do not have 24-hour periodicity. 
Figure 5

Particulate sucrose (left) and trehalose (right) measured as pmol L-1 in seawater (top), fmol cell-1 (middle) of Crocosphaera and Procholorococcus for trehalose and sucrose, respectively, and $\mathrm{mg}$ $\mathrm{g}^{-1}$ cell carbon (bottom) of Crocosphaera and Procholorococcus for trehalose and sucrose, respectively. The light grey vertical shading represents nighttime. The green box in the middleleft panel indicates the range of cellular sucrose quotas measured in Procholorococcus MIT1314 harvested mid-day in exponential growth. The blue points in the middle-right panel indicate the dawn and dusk values measured for trehalose quotas in Crocosphaera watsonii WH8501. In the top panels, the error bars represent one standard deviation around the mean value, including uncertainty from the quantification regression. The error bars in the middle panels represent one standard deviation around the mean. The error bars in the bottom panels represent the 95\% confidence interval given the confidence in the biomass quantification from underway flow cytometry.

A) Diel metabolite concentrations (peak area $\mathrm{L}^{-1}$, proportional to nmol $\mathrm{L}^{-1}$ ) of methionine cycle compounds, methylthioadenosine, and osmolytes. Error bars are the standard deviation of biological triplicates. The light grey vertical shading represents nighttime. B) Left: Time of peak abundance of diel transcripts related to the production or use of select diel osmolytes and primary metabolites. Fill color indicates the phylogenetic lineage of the transcript; outline color indicates whether the transcript is associated with production or consumption of the metabolite. Time of metabolite peak concentration $\left(\mathrm{nmol} \mathrm{L}{ }^{-1}\right)$ is in black. Right: Proportion of all transcripts and diel transcripts belonging to each taxon. $*=$ does not include select subgroups shown otherwise. Multivariate analyses based on $z$-scored particulate metabolite concentration (proportional to nmol L $\left.{ }^{-1}\right)$. A) NMDS of the first sampling period alone: Jul-26 ${ }^{\text {th }}-J u l 30$ th. The NMDS analysis results were significant (Monte Carlo randomization $p<0.01$ ) with a stress value of 0.18 . B) Within and between group variability from ANOSIM analysis using $z$-score standardized particulate concentrations of all metabolites $\left(\mathrm{nmol} \mathrm{L}^{-1}\right)$ from the first sampling period $(R=0.194$, $p<0.001)$. C) NMDS of the second sampling period alone: Jul 31st - Aug 3rd. The NMDS analysis results were significant (Monte Carlo randomization $p<0.01$ ) with a stress value of 0.17. D) NMDS of full dataset: Jul-26th - Aug-3rd. Colors indicate time of day that the samples were collected. The NMDS analysis results were significant (Monte Carlo randomization $p<$ 0.01 ) with a stress value of 0.18 . indicates the phylogenetic lineage of the transcript. Left: Peak time of transcript abundance or particulate metabolite concentration $\left(\mathrm{nmol} \mathrm{L}^{-1}\right)$. Right: Proportion of diel transcripts belonging to each taxa and proportion of all transcripts, regardless of diel oscillation, related to each metabolite belonging to each taxa.

859 Offset time (in hours) between the diel compounds and diel eukaryotic transcripts (top) or diel 
860 prokaryotic transcripts (bottom) that use or produce them. Diel significance of compounds was 861 based on the first sampling period, diel significance of eukaryotic transcripts was based on the first 862 sampling period, diel significance of the prokaryotic transcripts was based on both sampling 863 periods (RAIN fdr-corrected $p<0.05$ ).

Supplemental Figure 4

Field and culture particulate trehalose concentrations normalized to Crocosphaera cell count. Field data (black points) show the average and standard deviation at each time point over the full sampling period. Lab cultures (green circles) represent the values for the cultures harvested at dawn and dusk. Variability in technical replicates (for dusk) and biological duplicates (for dawn) are smaller than the points.

871

872

Supplemental Figure 5

Field and culture sucrose per cell Prochlorococcus. Field data (black points) show the median and range at each time point. The green box shows the maximum and minimum values of sucrose in triplicate axenic cultures of Prochlorococcus MIT1314 harvested at mid-day in exponential growth.

Time of day that compounds peak in the second sampling period.

Supplemental Table S1

882

883

Transcriptomes used to supplement the MarineRefII reference database (http://roseobase.org/data/).

\section{Supplemental Table S3}

Metabolites measured in this analysis. The average fold change from peak to trough, the maximum and minimum estimated or absolutely quantified values (pmol L-1), and whether the compound oscillates with 24-hour periodicity when normalized to volume of seawater filtered (water), when normalized to POC (POC), both (Both), or neither (None) for the first sampling period analyzed independently, second sampling period analyzed independently, and full dataset. The time of peak concentration for these various normalizations and time periods are provided in the final columns, rounded to the nearest hour. The net flux through the particulate pool calculated by the mean daily swing from max to minimum. * indicates metabolites for which samples 21-24 are removed and for which 6 samples in the second diel sampling period maybe affected by internal standard adjustments. + indicates metabolites for which 4 samples in the second sampling period might affected by IS adjustments. $\dagger$ notes that concentrations for DMSP are likely underestimates, as described in the methods.

\section{Supplemental Table S4}

Average and standard deviation of targeted metabolite composition at dawn (06:00) and dusk (18:00) from July $26^{\text {th }}-$ July $28^{\text {th }}$ ( $n=9$ for each time point), as the estimated particulate metabolite concentration, the percent of particulate organic carbon, and the percent of the particulate nitrogen. 
Supplemental Table S5

908

Pairwise comparisons of samples collected at different time points from the multivariate analyses

909 of particulate metabolite concentration during the first sampling period.

910

\section{Supplemental Table S6}

912

Prokaryotic transcripts that matched metabolites identified by organism taxa and KEGG

913 ortholog. If the transcript is significantly diel (RAIN fdr-corrected $p$-value $<0.05$ ) the time of

914

915 peak transcript abundance is provided ( $0 / 24$ is midnight, 12 is noon).

Eukaryotic transcripts that matched metabolites identified by organism taxa and KEGG ortholog. If the transcript is significantly diel (RAIN fdr-corrected $p$-value $<0.05$ ) the time of peak transcript abundance is provided ( $0 / 24$ is midnight, 12 is noon).

922 Particulate metabolite concentrations in normalized peak area per L of seawater filtered. Across a 923 single metabolite these values are proportional to molar concentration. Values should not be 924 quantitatively compared between two metabolites, since the ionization efficiency and matrix 925 effects influence different metabolites differently such that the same concentration can result in 926 difference in peak area. 


\section{Tables}

931 Table 1

932 Wind speed and surface mixed layer physical and biological variables over the two sampling 933 periods. Salinity, temperature, and dissolved oxygen (corrected with bottle measurements) are 934 from the CTD between 13-17 m. N+N and heterotrophic bacteria abundance are measured from 935 discrete samples at $15 \mathrm{~m}$. Particulate organic carbon (from underway beam attenuation), particulate 936 nitrogen, Prochlorococcus, Synechococcus, photosynthetic picoeukaryotes, and Crocosphaera are 937 measured from the ship-underway water intake near $7 \mathrm{~m}$.

938

\begin{tabular}{|c|c|c|}
\hline & $\begin{array}{l}\text { First Sampling period } \\
\text { Average } \pm S D(n)\end{array}$ & $\begin{array}{c}\text { Second Sampling period } \\
\text { Average } \pm S D(n)\end{array}$ \\
\hline Wind speed (kts) & $10.59 \pm 1.97(83)$ & $15.15 \pm 1.76(72)$ \\
\hline Mixed layer depth (m) & $20.92 \pm 5.20(25)$ & $36.12 \pm 6.42(19)$ \\
\hline Salinity & $35.38 \pm 0.01(62)$ & $35.39 \pm 0.00(33)$ \\
\hline Temperature $\left({ }^{\circ} \mathrm{C}\right)$ & $26.81 \pm 0.10(268)$ & $26.86 \pm 0.07(80)$ \\
\hline Nitrate + Nitrite (nM) & $3.02 \pm 0.90(19)$ & $3.34 \pm 1.65(9)$ \\
\hline Dissolved oxygen $(\mu \mathrm{mol} / \mathrm{kg})$ & $205.74 \pm 0.70(63)$ & $205.62 \pm 0.43(39)$ \\
\hline Particulate organic carbon $\left(\mu \mathrm{g} \mathrm{C} 1^{-1}\right)$ & $42.28 \pm 2.72(94)$ & $40.70 \pm 3.83(73)$ \\
\hline Particulate Nitrogen $\left(\mu \mathrm{mol} \mathrm{N} \mathrm{^{-1 } )}\right.$ & $0.44 \pm 0.04(18)$ & $0.40 \pm 0.03(12)$ \\
\hline $\begin{array}{l}\text { Heterotrophic bacteria abundance }\left(10^{6}\right. \\
\left.\text { cells } 1^{-1}\right)\end{array}$ & $508.3 \pm 27.3(22)$ & $534.1 \pm 30.8(47)$ \\
\hline Prochlorococcus abundance $\left(10^{6}\right.$ cells $\left.1^{-1}\right)$ & $161.23 \pm 11.75(98)$ & $196.38 \pm 15.37(55)$ \\
\hline Synechococcus abundance $\left(10^{6}\right.$ cells $\left.1^{-1}\right)$ & $0.85 \pm 0.07(98)$ & $0.89 \pm 0.06(55)$ \\
\hline $\begin{array}{l}\text { Photosynthetic picoeukaryote abundance } \\
\left(10^{6} \text { cells }^{-1}\right)\end{array}$ & $0.97 \pm 0.11(98)$ & $1.10 \pm 0.33(55)$ \\
\hline Crocosphaera abundance $\left(10^{6}\right.$ cells $\left.1^{-1}\right)$ & $0.16 \pm 0.06(98)$ & $0.31 \pm 0.07(55)$ \\
\hline
\end{tabular}




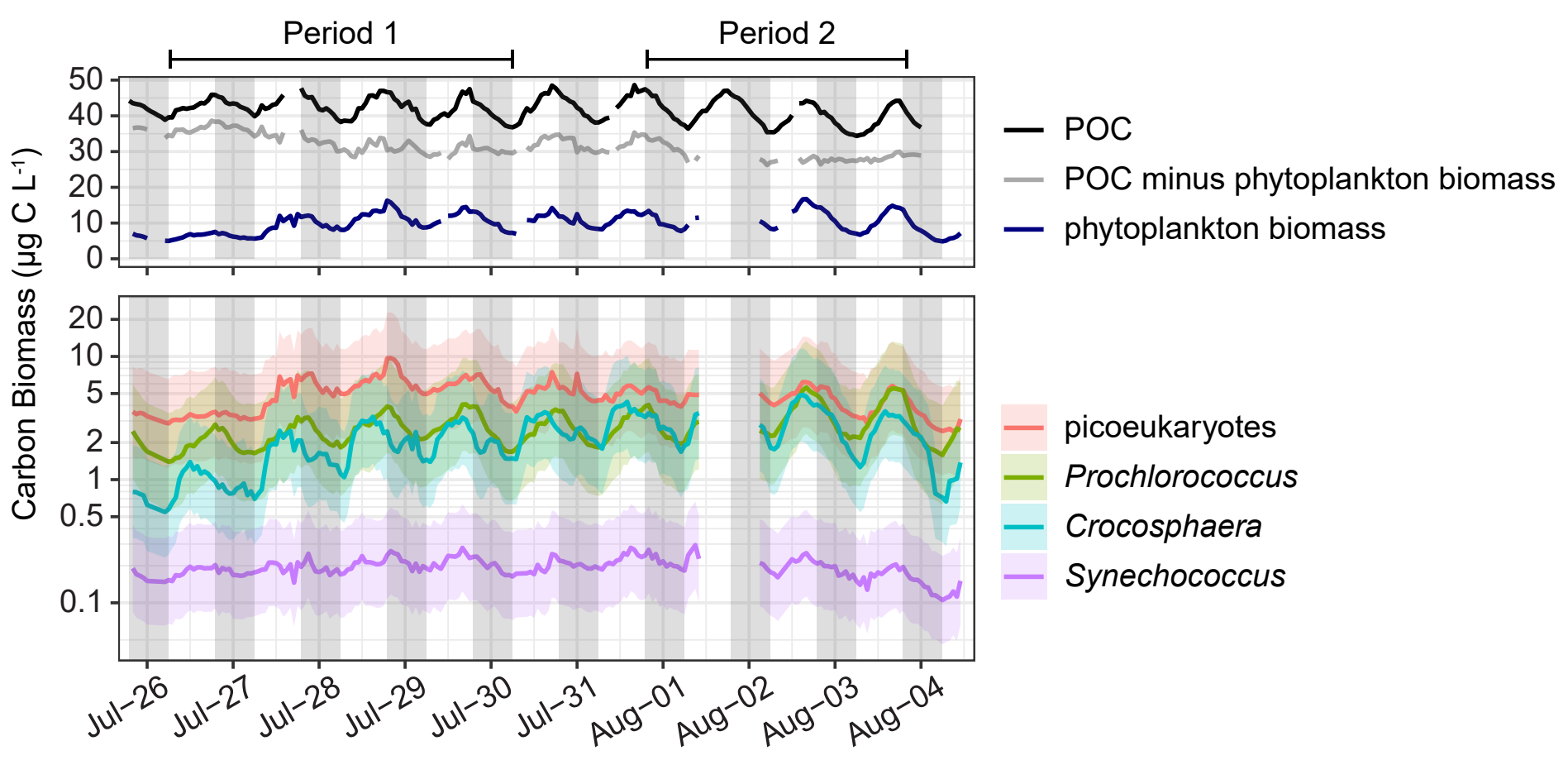

Figure 1. Top: Hourly averages of $P O C$ from beam attenuation (black line), total phytoplankton carbon biomass from flow cytometry (phytoplankton biomass, navy line), and the difference between the two (grey line). Bottom: Hourly averages of population specific carbon biomass of Prochlorococcus, Synechococcus, Crocosphaera, and photosynthetic picoeukaryotes (defined here as 2-4 $\mu \mathrm{m}$ ) from flow cytometry, with shaded area representing the $95 \%$ confidence interval. Breaks in the lines are due to short periods of instrument malfunction. The two sampling periods referred to in the text are indicated above the figure. 


\section{A. Mean nM}

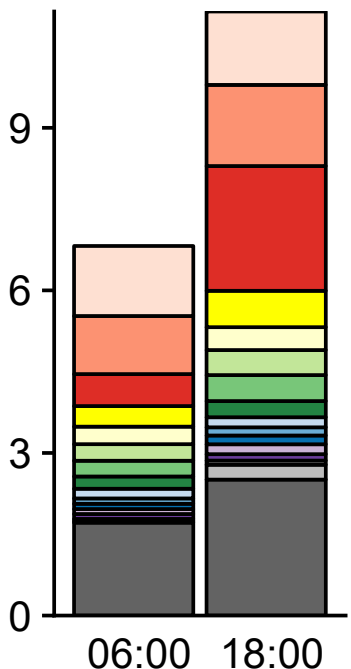

B. \% POC

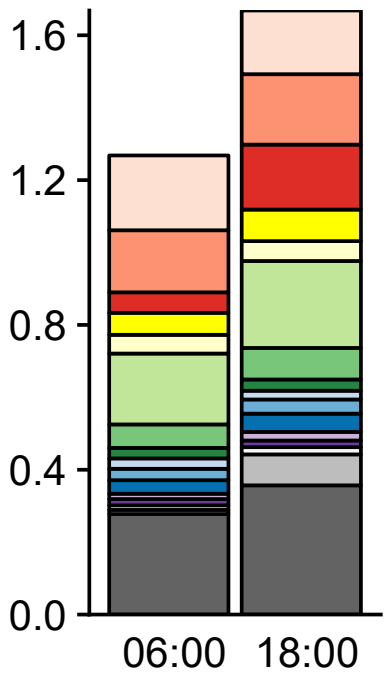

Time
C. $\% \mathrm{PN}$

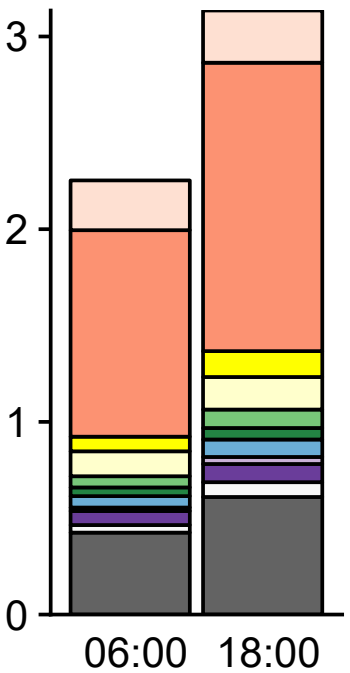

06:00 18:00

\section{$\square$ Glutamic acid Guanine DHPS \\ $\square$ Glutamine Arachidonic acid Homarine Aspartic acid

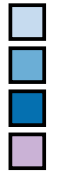 \\ DMSP \\ Glutathione Sucrose Choline \\ Arginine Adenosine Trehalose Other}

Figure 2. Average targeted metabolite composition at dawn (06:00) and dusk (18:00) from July 26th - July 28th ( $n=9$ for each time point), shown as the estimated particulate metabolite concentration (A), the percent of particulate organic carbon (B), and the percent of the particulate nitrogen (C). "Other" contains the sum of the rest of the metabolites (64 compounds). Osmolytes are in bold. Note the different y-axis scales. 
A.

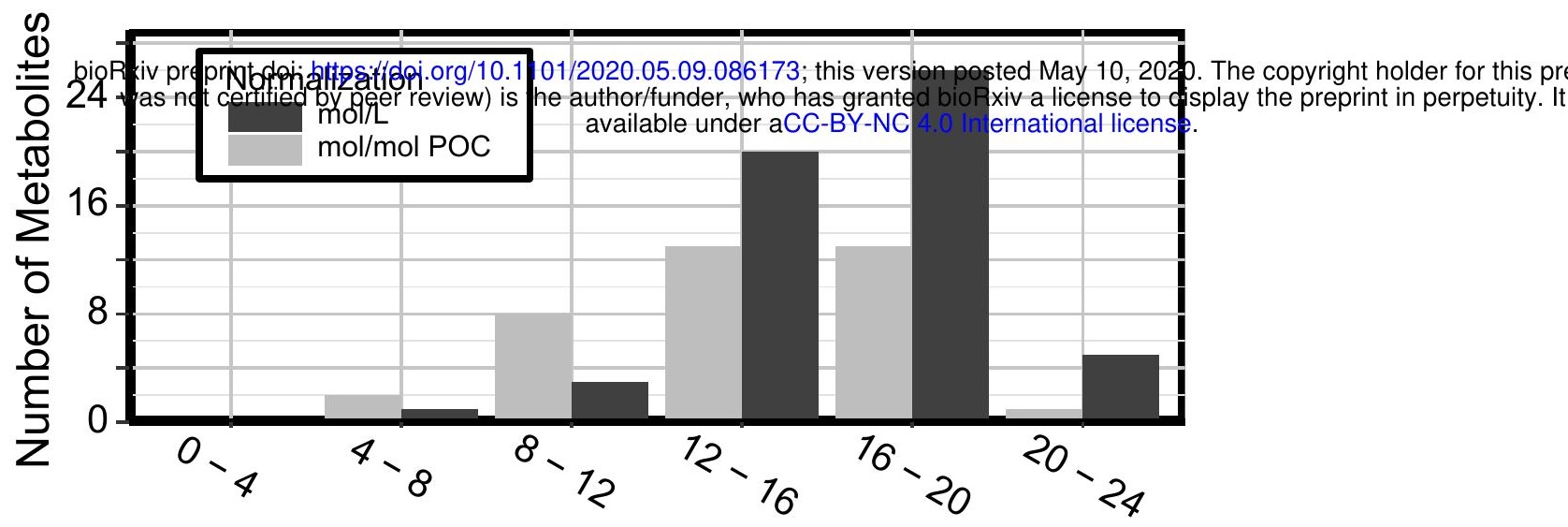

Time of Peak Metabolite Concentration

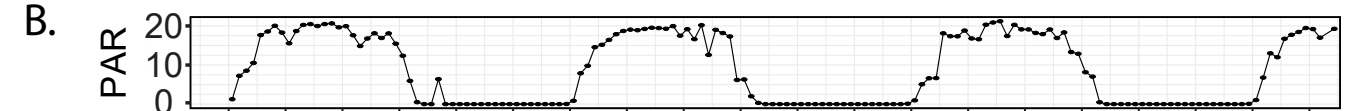

C.

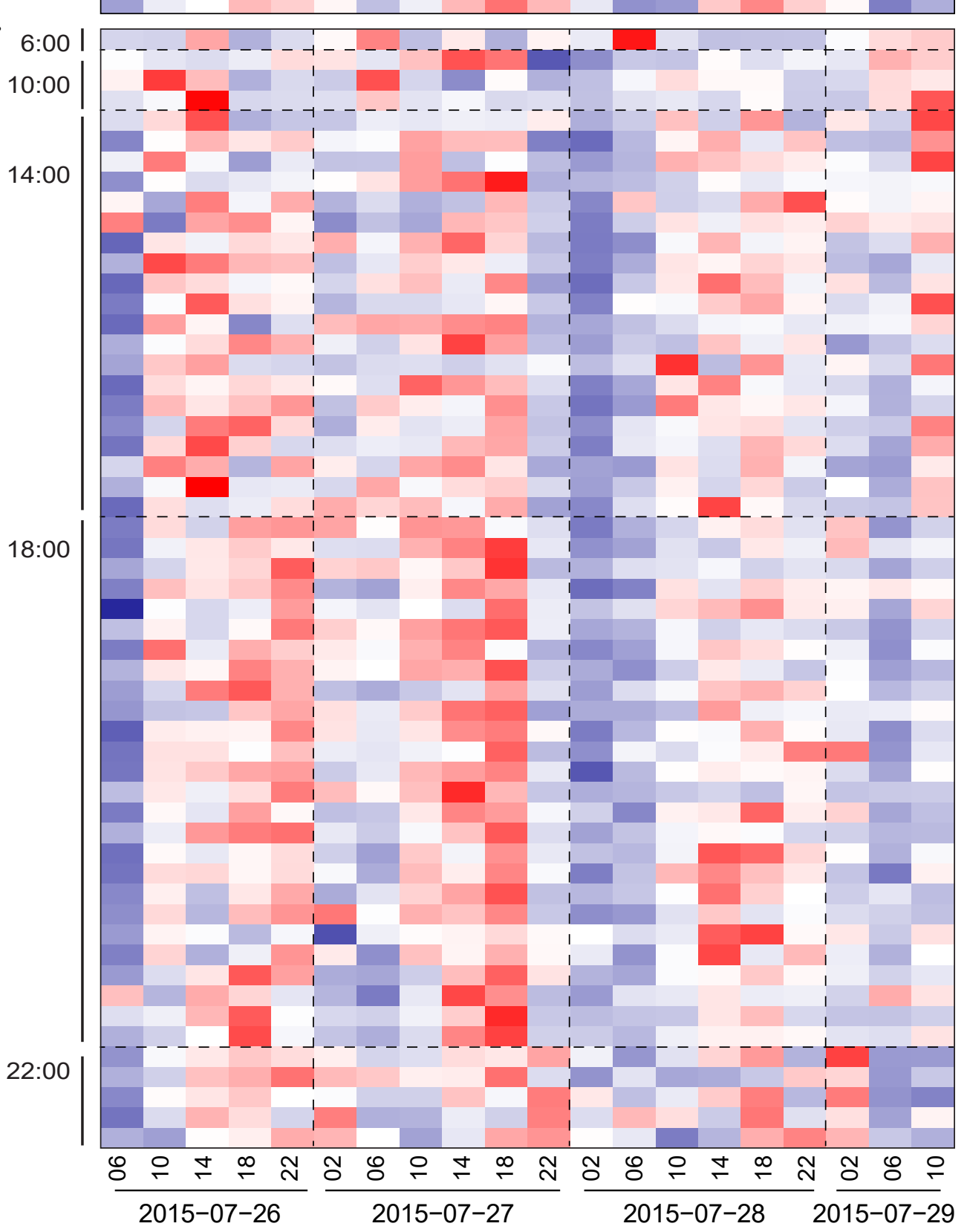

POC

Vanillic acid

Ergosterol

Pantothenate
Serine

Alanine

Asparagine

Chitobiose

Citric acid

Creatine

Glutathione

Homarine

AA

soleucine-Leucine

Lysine

Methylthioadenosine

Proline

S-adenosyl homocysteine

Taurine

Tryptophan

Tyrosine

UP-glucosamine

Valine

Xanthine

Adenine

Adenosine

Aminobutyric acid

AMP

Choline

Cystathionine

Çytidine

Cytosine

Gluconic acid

Glucosylglycero

Glutamine

Glycine betaine

GMP

Guanosine

sethionic acid

Methionine

Niacin

Phosphoglyceric acid

Pyridoxal Phosphate

Riboflavin

Ribose 5 phosphate

S-adenosyl methionine

trans Retinal

Trehalose

UDP-glucose

Arachidonic acid

Aspartic acid

EPA

henylalanine

Sucrose

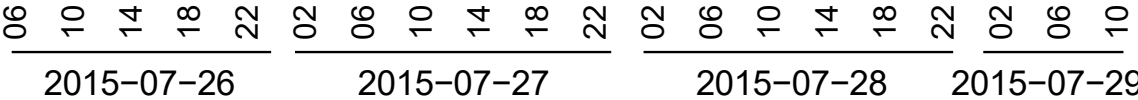

z-score of concentration

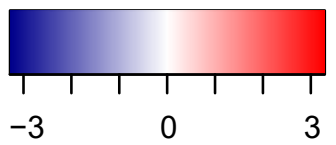

Figure 3. Time of day that significantly diel compounds peak in the first sampling period (A). Surface light (photosynthetically active radiation, PAR, $x 10 \mathrm{nmol}$ photon $\mathrm{m}-2 \mathrm{~s}-1)$ (B). Heat map showing the $\mathrm{z}$-score standardized concentrations of POC and of metabolites (nmol L-1) determined to be significantly diel in the first sampling period, arranged by time of peak concentration (C). 


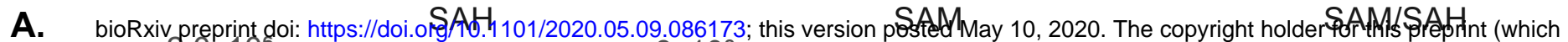
was noxcedffifed by peer review) is the authorffund dix wao has granted bigRxiv a license to display the preprint in perpetuity. It is made
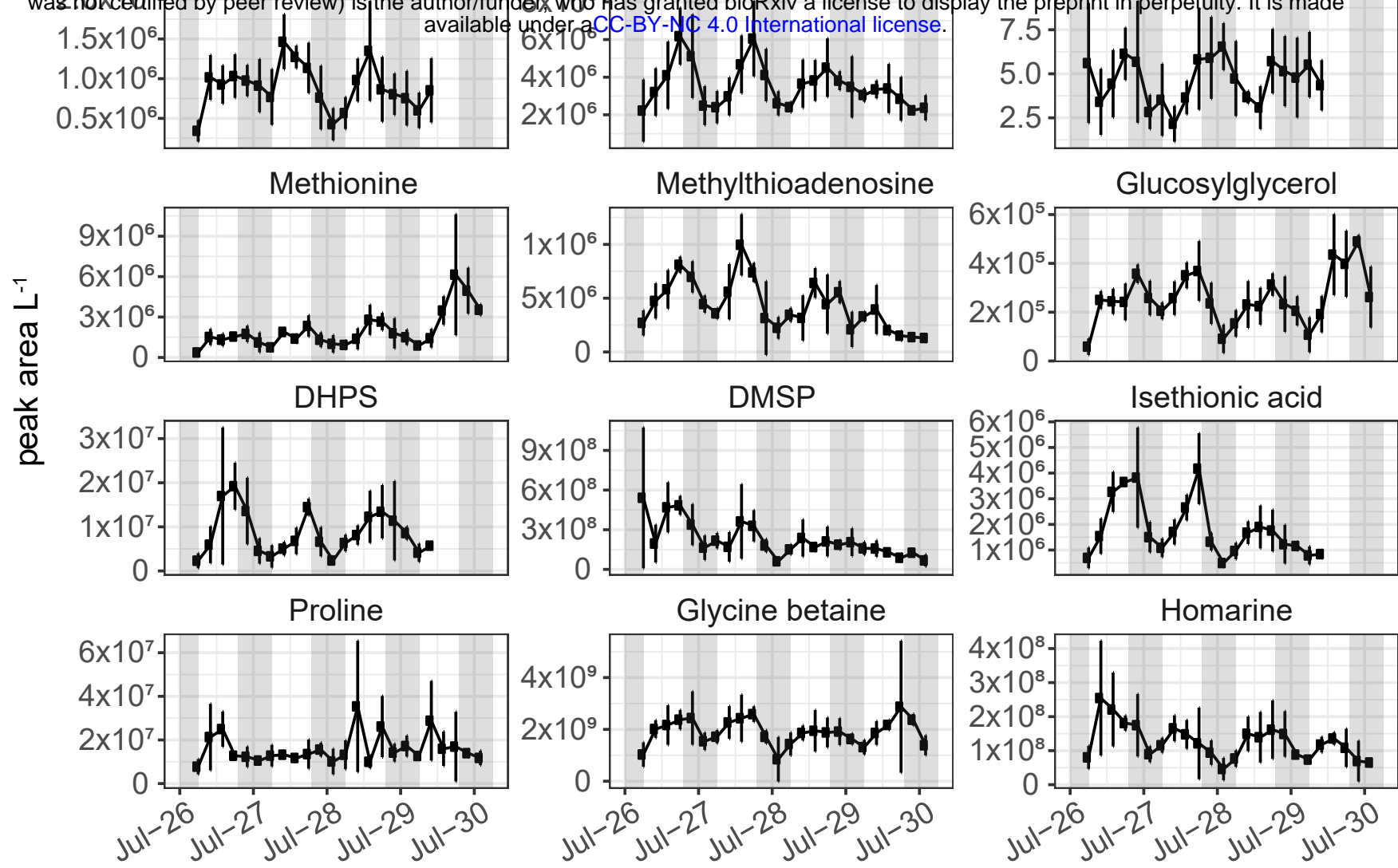

B.

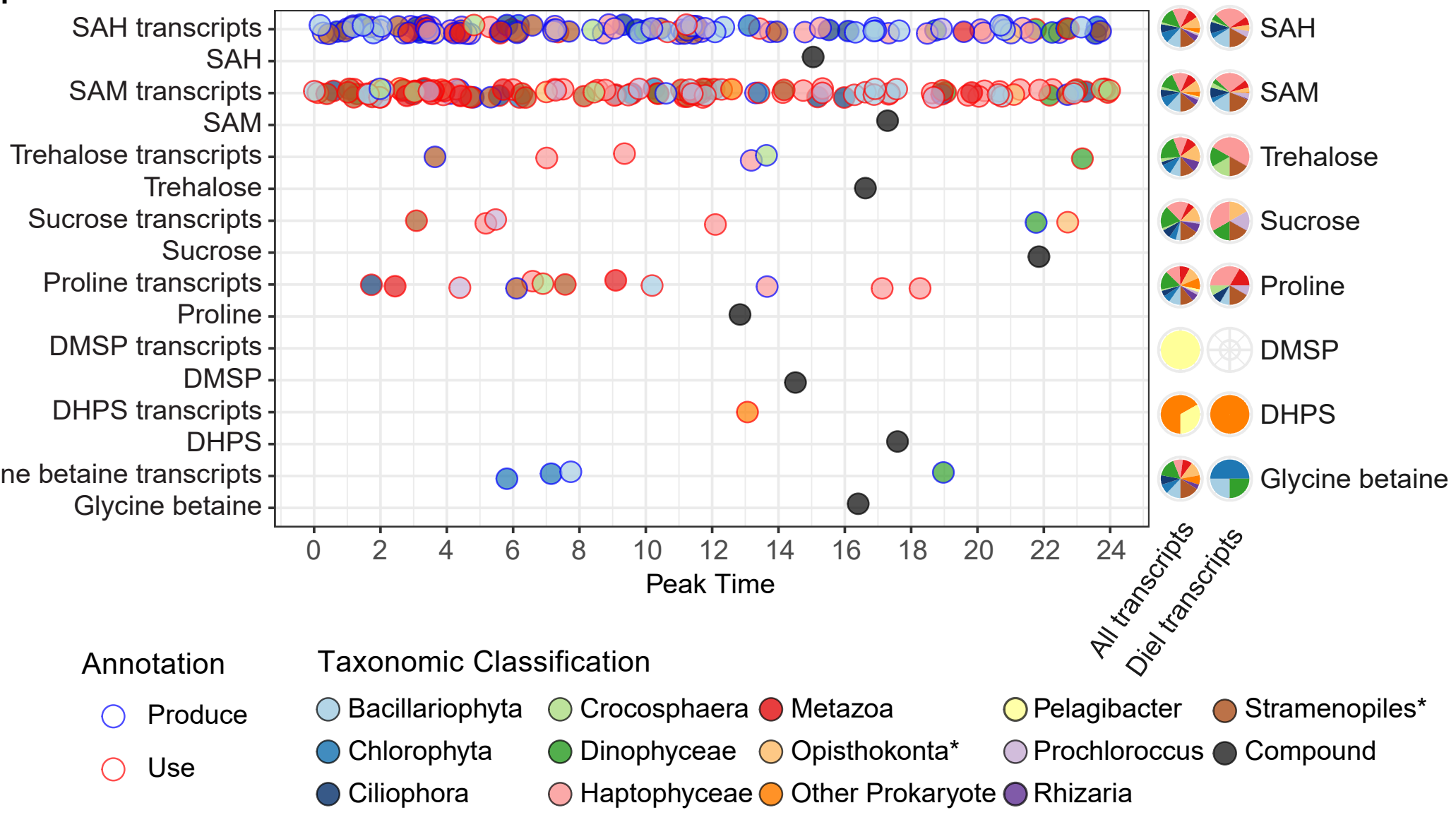

Figure 6: A) Diel metabolite concentrations (peak area L-1, proportional to nmol L-1) of methionine cycle compounds, methylthioadenosine, and osmolytes. Error bars are the standard deviation of biological triplicates. The light grey vertical shading represents nighttime. B) Left: Time of peak abundance of diel transcripts related to the production or use of select diel osmolytes and primary metabolites. Fill color indicates the phylogenetic lineage of the transcript; outline color indicates whether the transcript is associated with production or consumption of the metabolite. Time of metabolite peak concentration (nmol L-1) is in black. Right: Proportion of all transcripts and diel transcripts belonging to each taxon. $*=$ does not include select subgroups shown otherwise. 